\title{
ESPC y estilos de aprendizaje en ingeniería de producción
}

\author{
Yineth Reyes-Quintero ${ }^{a}$ \& Vlakxmir Robles-Marín \\ ${ }^{a}$ Maestría en Educación, Universidad Autónoma de Bucaramanga, Bucaramanga Colombia. yineth.reyes@unab.edu.co
${ }^{b}$ Escuela de Ingeniería de Producción, Instituto Universitario de la Paz, Barrancabermeja, Colombia. vlakxmir.robles@unipaz.edu.co
}

Resumen - Se diseñó una estrategia partiendo del estilo dominante de aprendizaje a través del estudio de situaciones problémicas contextualizadas (ESPC), para la enseñanza del concepto calidad. Participaron 30 estudiantes de primer ingreso al programa de Ingeniería de Producción pertenecientes a la jornada nocturna del periodo 2016. Los resultados mostraron un impacto positivo en el rendimiento académico del grupo objetivo frente al grupo experimental. La diferencia de los promedios aritméticos en las notas obtenidas por los grupos fue significativa.

Palabras Clave — Estilo de aprendizaje, estrategias de enseñanza, estrategias de aprendizaje, rendimiento académico, ingeniería, calidad, ESPC

Recibido: 20 de enero de 2017. Revisado: 20 abril de de 2017. Aceptado: 10 de mayo de 2017.

\section{Learning style and ESPC in production engineering students}

\begin{abstract}
It was designed a strategic starting of dominant style of learning through the Studies of Contextualized Problem Situations (ESPC, for its acronym in Spanish), to teach the concept of quality. Participated 30 first semester students from the Production Engineering program of the night class of the 2016 period. The results show a positive impact on the academic achievement of the target group compared to the experimental group. The difference of the arithmetic mean in the scores obtained by the groups were significant.
\end{abstract}

Keywords - Learning style, teaching strategies, learning strategies academic achievement, engineering, quality, ESPC

\section{Introducción}

Los estilos de aprendizaje son abordados conceptualmente por un gran número de autores. Felder y Hernández los definieron como "los modos en que el individuo característicamente adquiere, retiene y recupera información” (1). Smith concuerda con la definición anterior en cuanto al modo por el cual un individuo procesa información y adicionalmente habla de lo que siente y cómo se comporta en las situaciones de aprendizaje (2) Según Cruickshank, Baimerm y Metcalf, "es un patrón de conducta y ejecución consistente por el cual obtenemos experiencias de aprender" (2). En conjunto se habla de lo referente al ser humano y su relación con el aprendizaje.

Estudios correlacionan el estilo de aprendizaje con múltiples variables. Khadija y Trali, relacionaron el estilo y las estrategias de aprendizaje. Partieron con el cuestionario VARK el análisis del grupo perteneciente a la facultad de anatomía. Los resultados obtenidos mostraron a Trimodal como el estilo preferente de aprendizaje. (3). Partiendo con instrumentos como el cuestionario CHAEA y el Time Managent Behavior Scale (TMBS-1994), los investigadores Durán-Aponte y Pujol correlacionaron el estilo de aprendizaje el rendimiento académico y la gestión del tiempo. Se encontró relación entre los estilos Teórico y Reflexivo y las dimensiones establecimiento de objetivos y prioridades y percepción de control de tiempo (4).

Ventura analizó la producción científica de los años 2000 al 2010, en torno a la interacción de los estilos de aprendizaje con las prácticas de enseñanza. Concluyó que si existe evidencia de esta relación (5). En Colombia, el Ministerio de Educación Nacional tomó las metodologías de enseñanza basadas en los estilos de aprendizaje, como una herramienta para el mejoramiento de la calidad académica y la permanencia estudiantil (6). Consecuencia de lo anterior la investigación tomó el estilo dominante de aprendizaje del grupo para diseñar una estrategia de enseñanza. Entendida esta última como, procedimientos que el docente utiliza en forma reflexiva y flexible para promover el logro de aprendizaje en el alumno. (7). Pero, ¿Qué estrategia de enseñanza trabajar?

Córchuelo propuso a través del Estudio de situaciones problémicas contextualizadas (ESPC), trabajar la educación del ingeniero entorno al mundo cotidiano. El ESPC comprende principalmente nueve actividades: Sensibilización; Contextualización; Exploración de Conceptos; Análisis de Relaciones; Diseño, simulación y/o experimentación; Análisis de Resultados; Socialización; Valoración y Ajustes y Nuevo Discurso (8). Por otra parte, los resultados de las investigaciones de Hernández y Cardona arrojaron la existencia de una relación positiva entre las variables rendimiento académico y estilo y estrategias de aprendizaje (9). Por lo anterior se esperó un impacto de la estrategia, en el rendimiento académico de los estudiantes. Entendido este último como la medición a través de la nota obtenida en las pruebas realizadas (10)

Según Moreira es necesario comprender conceptos para comprender el mundo y desarrollarnos cognitivamente (11). El Ingeniero de Producción presenta un perfil ocupacional y profesional que, le exige un conocimiento adecuado para el manejo de la calidad en procesos relacionados con bienes y 
servicios. La calidad tiene un gran número de definiciones, dependiendo del autor o la organización y su enfoque. Esto dificulta la comprensión del mismo y por consecuencia su aplicación. Para esta investigación se utilizó el estilo de aprendizaje dominante y los preconceptos del tema calidad como insumo para el diseño de una estrategia que impactó en el rendimiento académico.

\section{Metodología}

Para el primer semestre del año 2016, se inscribieron un total de 80 estudiantes al programa ingeniería de producción. De esta población, se abordaron 30 discentes que contaban con mayoría de edad y firmaron autorización para la participación en el ejercicio. El total de la población se encontró inscrita para la jornada nocturna, distribuidos así: género femenino $27 \%$ y género masculino $73 \%$. La institución ofertó dos cursos de primer ingreso para la jornada nocturna, por lo cual se trabajó con un grupo experimental y un grupo de control. El primero estuvo compuesto por seis mujeres y diez hombres para un total de dieciséis personas. El segundo grupo estuvo compuesto por dos mujeres y doce hombres para un total de catorce personas.

Para determinar el estilo dominante del grupo se utilizó el instrumento Index of Learning Styles (ILS). Está constituido por 44 preguntas desarrolladas por Felder y Soloman en 1993. El objetivo principal de la prueba, es conocer las preferencias entorno al estilo de aprendizaje según el modelo de Felder y Silverman.

El modelo bipolar de Felder y Silverman (12) consta de 4 dimensiones y 5 categorías por dimensión:

- Dimensión de Procesamiento: Los perfiles de esta dimensión son Muy Activo, Activo, Neutral, Reflexivo y Muy Reflexivo.

- Dimensión de Percepción: Los perfiles de esta dimensión son Muy sensitivo, Sensitivo, Neutral, Intuitivo y Muy Intuitivo.

- Dimensión de Recepción: Los perfiles de esta dimensión son Muy Visual, Visual, Neutral, Verbal y Muy Verbal.

- Dimensión de Progreso: Los perfiles de esta dimensión son Muy Secuencial, Secuencial, Neutral, Global y Muy Global. Felder y Soloman propusieron una serie de actividades dependiendo del estilo, que ayudan a mejorar el aprendizaje (13). Barriga y Hernández indicaron que las estrategias tanto de aprendizaje como de enseñanza, deben considerarse como complementarias y no excluyentes una de la otra (14). Entorno a estos exponentes se realiza la recopilación de sus teorías ver. Anexo 1. Esta presenta desde una óptica constructivista las estrategias que se sugieren utilizar de acuerdo al estilo de aprendizaje dominante del grupo.

Los resultados se obtienen por dimensión en tres tendencias: Fuerte, Moderado y Equilibrado. La tendencia Fuerte y Moderado se inclinan hacia el lado positivo o negativo de la escala según la tabulación de las respuestas. Esta inclinación se asocia a cuatro perfiles por dimensión. En la Tabla 1 se puede observar los rangos para cada perfil.
Tabla 1

Tendencias y Perfiles según modelo de Felder para el análisis de los datos recopilados con el instrumento ILS.

\begin{tabular}{lllllll}
\hline Tendencia & Fuerte & Mod & Equilibrado & Mod & Fuerte \\
\hline Dimensión & 11 Y 9 & 7 Y 5 & $\begin{array}{l}3 \text { Y } \\
1\end{array}$ & $\begin{array}{l}-1 \\
\text { Y }\end{array}$ & -5 Y -7 & -9 Y -11 \\
& & & & & \\
\hline $\begin{array}{l}\text { D1 } \\
\text { (Procesamiento) }\end{array}$ & $\begin{array}{l}\text { activo } \\
\text { D2 }\end{array}$ & activo & neutral & reflexivo & $\begin{array}{l}\text { muy } \\
\text { reflexivo }\end{array}$ \\
\hline $\begin{array}{l}\text { (Percepción) } \\
\text { D3 }\end{array}$ & $\begin{array}{l}\text { sensitivo } \\
\text { (Recepción) }\end{array}$ & sensitivo & neutral & intuitivo & $\begin{array}{l}\text { muy } \\
\text { intuitivo }\end{array}$ \\
\hline $\begin{array}{l}\text { D4 } \\
\text { (Progreso) }\end{array}$ & $\begin{array}{l}\text { muy } \\
\text { secuencial }\end{array}$ & visual & necutral & verbal & $\begin{array}{l}\text { muy } \\
\text { verbal }\end{array}$ \\
\hline
\end{tabular}

Fuente: Autores.

La Tabla 1 está compuesta por 6 columnas y 6 filas. Se enuncia por cada tendencia (Fuerte, Moderado o Equilibrado) cual es el perfil del discente (Activo/Reflexivo, Sensitivo/Intuitivo, Visual/Verbal y Secuencial/Global) según el rango numérico (-11 y 11) obtenido por este, en cada dimensión.

Los preconceptos del tema calidad se abordaron a través de un test compuesto por once preguntas. Se tomó el concepto de calidad dado por la organización internacional de normalización (ISO). "Grado en el que un conjunto de características (rasgo diferenciador) inherentes a un objeto (entidad, ítem, cualquier cosa que pueda percibirse o concebirse) cumple con los requisitos (necesidad o expectativa establecida generalmente implícita u obligatoria)" (15)

Después del diagnóstico del estilo de aprendizaje y el concepto calidad, se diseñó la estrategia entorno al ESPC. Esta se aplicó al grupo experimental. El grupo control recibió la clase con la estrategia usada normalmente por el docente. Al final de la implementación se procedió a la aplicación nuevamente del test sobre el concepto calidad. Se comparó el promedio aritmético de las notas obtenidas en la pre y pos prueba para los dos grupos. Los análisis estadísticos incluyendo los de fiabilidad, se realizaron a través del programa IBM SPSS Statistics 21.

\section{Resultados}

Los resultados del proceso se abordarán en tres secciones: Diagnóstico del estilo dominante de aprendizaje, Diagnóstico de los conocimientos del concepto calidad y el impacto de la estrategia. En la Tabla 2 se observan los puntajes obtenidos al realizar los análisis descriptivos para los datos recopilados en el diagnóstico del estilo de aprendizaje. Las medidas de tendencia central son congruentes entre sí, arrojando como tendencia equilibrado para las cuatro dimensiones. El coeficiente de variación para la dimensión 1 evidencia la estabilidad de estos datos, para las dimensiones 2, 3 y 4 los valores son muy variables dados los coeficientes obtenidos.

Tabla 2

Análisis descriptivos para los datos recolectados.

\begin{tabular}{lcccc}
\hline & D1 & D2 & D3 & D4 \\
\hline Media & 2,7 & 2,7 & 2,6 & 2,7 \\
\hline Mediana & 3 & 3 & 3 & 3 \\
\hline Moda & 3 & 3 & 3 & 3 \\
\hline Desviación Estándar & 0,5 & 0,6 & 0,8 & 0,7 \\
\hline Coeficiente de Variación & 19,4 & 21,7 & 31,3 & 25,2 \\
\hline Fuente: Autores & & & &
\end{tabular}


Tabla 3.

Análisis descriptivos para los datos recolectados.

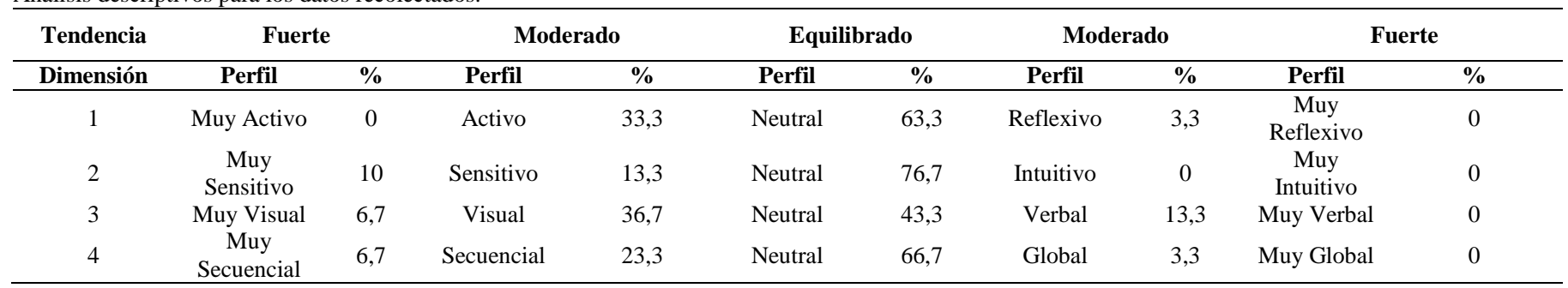

Fuente: Autores.

Al organizar el conjunto de datos obtenidos por el ILS, teniendo en cuenta la Tabla 1 , se genera una matriz compuesta por 11 columnas y 7 filas como se puede observar en la Tabla 3. La primera columna enuncia las dimensiones, en las columnas 2, 4, 6, 8 y 10 se presentan los perfiles por cada dimensión. Las columnas 3, 5, 7, 9 y 11 indican el porcentaje de discentes que pertenecen a cada perfil. Al observar esta tabla se encontró un gran número de estudiantes con tendencia Equilibrado y perfil Neutral. A este resultado le sigue la tendencia Moderado hacia el lado positivo de la escala, comprendiendo los perfiles Activo y Visual como los más representativos. La tendencia fuerte hacia el lado negativo de la escala no es representativa para ninguna dimensión.

$\mathrm{Al}$ analizar las dimensiones se encontró que: La dimensión de procesamiento presentó el 63,3\% de los estudiantes tienden a procesar la información de forma neutral, un 33,3\% lo hace de manera activa y solo el 3,3\% es reflexivo; al momento de percibir la información, un 76,7\% de la población lo hace de forma neutral, un $13,3 \%$ lo hace de forma sensitiva y un $10 \%$ la percibe muy sensitivamente; el 43,3\% recepciona la información externa de forma neutral, el 36,7\% lo hace de manera visual, el 13,3 \% verbal y solo un 6,7 \% es muy visual. Siendo esta la dimensión más variada; Al momento de conocer el progreso del estudiante hacia el entendimiento, el 66,7 \% de los estudiantes es neutral, el 23,3\% es secuencial y el 3,3 \% es global. El diagnóstico indicó que, la mayor concentración de la población abordada se encontró en la tendencia equilibrado perfil neutral. Sin embargo no se desconoció la tendencia hacia los lados positivos y negativos de la escala en el diseño de la estrategia.

Los resultados del test de preconceptos presentó un Kuder Richardson (16) de 0,720. La validez del instrumento se abordó con el juicio de expertos. El alfa de Cronbach para el juicio fue de 0,756. En ambos casos se analizaron once elementos. El grupo experimental obtuvo un promedio aritmético de dos punto cinco sobre cinco para la pre prueba. El grupo control obtuvo un promedio de dos punto siete sobre cinco. La diferencia entre grupos para la prueba diagnóstico fue de cero punto dos. Lo que permitió inferir que, los estudiantes no contaban con conocimientos claros sobre el tema calidad. Dado que a nivel cuantitativo para aprobar el test se debió obtener una nota igual o mayor de tres, fue correcto afirmar que ninguno de los dos grupos aprobó la prueba.

La pregunta con menor número de aciertos fue la relacionada con la definición dada por ISO al termino calidad. Las preguntas con mayores aciertos fueron: la relacionada en establecer como las organizaciones conocen lo que sus clientes necesitan; y la relacionada con limitación del termino calidad a producto.

La estrategia partió desde las propias necesidades de los estudiantes. Se enfocó hacia la calidad del servicio ofrecido en la institución y como el ingeniero de producción puede mejorar esté. Se estudió la importancia de la institución y su impacto en la región; las relaciones entre necesidades del cliente, producto/servicio y satisfacción del cliente; y se delimitó la situación problema según los recursos disponibles. Se diseñó un folleto informativo, una actividad lúdica y un taller con la comunidad estudiantil. Se generó una cartilla comunicando la experiencia.

$\mathrm{Al}$ culminar la estrategia se procedió a la aplicación del pos test. Se siguieron los parámetros definidos para la aplicación de instrumentos y se aplicó el mismo día para los dos grupos. El alfa de Cronbach para los datos obtenidos en el pos prueba fue de 0,719. El grupo experimental presentó un promedio aritmético de tres punto ocho sobre cinco, en las notas del pos test. Por su parte el grupo control obtuvo un promedio aritmético de dos punto cero sobre cinco.

Las notas obtenidas por los grupos en el pre y pos test se relacionan en la Tabla 4. En ella se observó que, para el post Test las notas tuvieron una variación considerable entre los grupos. El grupo experimental obtuvo un promedio aritmético de tres punto ocho y el grupo control tuvo un promedio aritmético de dos punto cero. La diferencia entre los grupos fue mayor a un punto.

Para correlacionar los datos se trabajó previamente la normalidad de los mismos. El grupo experimental presentó estadístico en la prueba de Kolmogorov-Smirnov de 0,222 y el grupo de control 0,308 respectivamente. Estos valores permitieron indicar normalidad para un nivel de significación inferior a 0,034 y 0,001. La distribución fue normal dado que los datos fueron mayores de 0,05 para todos los casos. (17).

Se realizó el análisis correlacional y de medias para establecer el impacto de la estrategia en el grupo. En el primero se obtuvo valores superiores a 0,5. Esto indicó una correlación positiva intensa entre las variables. Cuando se analizó la media, el grupo experimental representado por el número uno, presentó una valoración mayor a la del grupo control. Los resultados se muestran en las Tablas 5 y 6 respectivamente.

Dado que el valor medio de las notas obtenido por el grupo experimental fue significativamente mayor que el valor obtenido por el grupo control $(p<0,01)$, se pudo afirmar que la estrategia tuvo un impacto positivo en el rendimiento académico. 
Tabla 4

Nota pre y pos prueba grupo experimental y control

\begin{tabular}{|c|c|c|c|c|c|c|c|c|c|c|c|c|c|c|c|c|c|}
\hline ID & & 337 & 771 & 912 & 553 & 365 & 957 & 897 & 114 & 115 & 165 & 964 & 293 & 757 & 134 & 512 & 748 \\
\hline IN & & 1 & 1 & 1 & 1 & 1 & 1 & 1 & 1 & 1 & 1 & 1 & 1 & 1 & 1 & 1 & 1 \\
\hline \multirow{2}{*}{$\begin{array}{c}\text { NOTA } \\
\text { PRUEBA }\end{array}$} & PRE & 3,6 & 3,6 & 2,1 & 0,0 & 2,1 & 2,1 & 3,6 & 5,0 & 2,1 & 3,6 & 2,9 & 2,9 & 2,1 & 2,9 & 2,1 & 0,0 \\
\hline & POS & 3,6 & 4,3 & 2,9 & 3,6 & 3,6 & 2,9 & 3,6 & 3,6 & 4,3 & 5,0 & 3,6 & 4,3 & 4,3 & 4,3 & 4,3 & 2,9 \\
\hline \multicolumn{2}{|c|}{ ID } & & 20 & 9219 & 377 & 219 & 120 & 60 & 445 & & 39 & 103 & 224 & 940 & 659 & 99 & 964 \\
\hline \multicolumn{2}{|c|}{ IN } & & 0 & 0 & 0 & 0 & 0 & 0 & 0 & & 0 & 0 & 0 & 0 & 0 & 0 & 0 \\
\hline NOTA & PRE & & 3,6 & 2,1 & 1,4 & 2,1 & 3,6 & 2,1 & 2,1 & &, 3 & 2,1 & 2,1 & 5,0 & 2,1 & 0,0 & 4,3 \\
\hline PRUEBA & POS & & 0,0 & 2,1 & 0,0 & 2,9 & 2,1 & 2,1 & 2,1 & & 1 & 3,6 & 2,1 & 4,3 & 2,9 & 0,0 & 2,1 \\
\hline
\end{tabular}

Fuente: Autores

Tabla 5

Resultados análisis correlacional.

\begin{tabular}{llll}
\hline \multicolumn{3}{c}{ Correlaciones } & \\
\hline \multirow{2}{*}{$\begin{array}{l}\text { Pos } \\
\text { prueba }\end{array}$} & Correlación de Pearson & 1 &, $685^{* *}$ \\
\cline { 2 - 4 } & Sig. (bilateral) &, 000 \\
\cline { 2 - 4 } Inter & $\mathrm{N}$ & 30 & 30 \\
\cline { 2 - 4 } & Correlación de Pearson &, $685^{* *}$ & 1 \\
\cline { 2 - 4 } & Sig. (bilateral) &, 000 & 30 \\
\cline { 2 - 3 } **. La correlación es significativa al nivel 0,01 (bilateral). &
\end{tabular}

Fuente: IBM SPSS Statistics 21

Tabla 6

Resultados de las Medias para los grupos.

\begin{tabular}{lllllll}
\hline \multicolumn{6}{c}{ Estadísticos de grupo } \\
\hline & & si 1 / no 0 & N & Media & $\begin{array}{c}\text { Desviación } \\
\text { típ. }\end{array}$ & $\begin{array}{c}\text { Error típ. de } \\
\text { la media }\end{array}$ \\
\hline posprueba & 1 & 16 & 3,8188 &, 61125 &, 15281 \\
\cline { 2 - 7 } & 0 & 14 & 2,0286 & 1,28687 &, 34393 \\
\hline
\end{tabular}

Fuente: IBM SPSS Statistics 21.

\section{Conclusiones}

El estilo dominante de aprendizaje según el inventario de estilos de aprendizaje (ILS) se encontró en la tendencia equilibrado perfil neutral.

La estrategia diseñada entorno al estilo dominante de aprendizaje tuvo un impacto positivo en el rendimiento académico del grupo abordado. Por tanto se recomienda caracterizar a los estudiantes del programa.

En futuras investigaciones se recomienda analizar el comportamiento de los estudiantes frente al docente del área.

La construcción de una herramienta que mida el desempeño docente en educación superior es importante. En Colombia, al momento de concluir esta investigación no se registró un instrumento definido para tal fin.

\section{Referencias}

[1] Bertel, P., Torres, P. y Díaz-Granados, F., Estilos y estrategias de aprendizaje en estudiantes de fonoaudiología. México, 2010.

[2] Gonzales-Clavero, M.V.,.Estilos de aprendizaje: Su influencia para aprender a aprender, 2011.

[3] Khadija, Z. and Trali, G., Learning styles-understanding for learning strategies. 2015.

[4] Durán-Aponte, E. y Pujol, L., Estilo de aprendizaje, gestión del tiempo y rendimiento académico en estudiantes universitarios. 2012.

[5] Estilos de Aprendizaje y prácticas de enseñanza en la universidad. Un binomio que sustenta la calidad educativa. Ventura, Ana. México: s.n.,
Vol. 33. 2011.

[6] Ministerio de Educación Nacional de la Republica de Colombia. Guía de Implementación del Modelo de Gestión de Permanencia y Graduación Estudiantil en Instituciones de Educación Superior. Bogotá - Colombia: Imprenta Nacional De Colombia, 2015.

[7] Barriga, Frida y Hernández, Gerardo. Estrategias docentes para un aprendizaje significativo. México: Mac Graw Hill, pág. 180. 2010.

[8] Corchuelo, Miguel. Savia. Un Giro en la Educación en Ingeniería. Popayán: Universidad del Cauca, 2007.

[9] Hernández, A. y Cardona, A., Estilos y estrategias de aprendizaje en el rendimiento académico de los alumnos del área de inglés de la licenciatura en lenguas modernas de la universidad de la salle. Bogotá: s.n., 2008.

[10] El Rendimiento Académico, concepto, investigación y desarrollo. EdelNavarro, R. s.l.: REICE: Revista Electrónica Iberoamericana sobre Calidad, Eficacia y Cambio en Educación, 2003.

[11] ¿Por qué conceptos? ¿Por qué aprendizaje significativo? ¿Por qué actividades colaborativas? ¿Por qué mapas conceptuales? Moreira, Marco. S.l.: Qurriculum: Revista de Teoría, investigación y práctica educativa, 2010.

[12] Learning and Teaching Styles in Engineering Education. Felder, Richard y Silverman, Linda. Engineering Education, págs. 674-681. 1988.

[13] Felder, R. y Soloman, B., Richard Felder`s Home Page. Learning Styles and Strategies. [En línea] [Citado el: 1 de 08 de 2016.] www4.ncsu.edu/unity/lockers/users/f/felder/public/ILSdir/Styles.htm.

[14] Barriga, F. y Hernández, G., Estrategias Docentes para un Aprendizaje Significativo. México: Mac Graw Hill, 2010.

[15] Organización Internacional de Estandarización. ISO 9000:2015 Sistemas de Gestión de Calidad Fundamentos y Vocabulario. Ginebra: s.n., 2015.

[16] Celina, O y Campo - Arias, A. Revista Colombiana de psiquiatría. Págs. 572-580. 2005.

[17] García Bellido, R, Gonzáles Such, J y Jornet Meliá, J. InnovaMide. Pruebas no Paramétricas. [En línea] 2010. https://www.google.com.co/url?sa=t\&source=web\&rct=j\&url=http:ww w.uv.es/innomide/spss/SPSS/SPSS_0802A.pdf\&ved=0ahUKEwjTmcS xotbQAhWGLyYKHbEWBnYQFghKMAw\&usg=AFQjCNHn.

Y.M. Reyes-Quintero, recibió el título de Ing. de Producción en el año 2010 por parte del Instituto Universitario de la Paz, Barrancabermeja, Colombia. MSc. en Sistemas Integrados de Gestión- HSEQ, de la Universidad Camilo José Cela, Madrid, España, para el año 2014. MSc. en Educación de la Universidad Autónoma de Bucaramanga, Bucaramanga, Colombia para el año 2017. Certificada como auditora interna para las normas ISO 9001, ISO $14001 \mathrm{y}$ OHSAS 18001 por Bureau Veritas. Laboró desde el 2010 al 2012, como Auxiliar HSE para el proyecto Ecodiesel en la planta de Biodiesel de la Gerencia Refinería Barrancabermeja, Barrancabermeja, Colombia. Ingresó como docente al Instituto Universitario de la Paz en el año 2013. Durante el año 2015 ocupó el cargo de asistente de vicerrectoría y actualmente continua laborando para la escuela de Ingeniería de Producción como delegada del proceso de acreditación y gestión de calidad.

ORCID: 0000-0003-3843-7753

V. Robles-Marín, obtuvo el título de Economista e Ing. Industrial de la Universidad Industrial de Santander, Bucaramanga, Colombia, en los años 2003 y 2011 respectivamente. Esp. en Educación Matemática para el año 2012 con la Universidad Industrial de Santander, Bucaramanga, Colombia. Con la Universidad EAFIT obtuvo el título de Esp. en Mantenimiento Industrial para el año 2016. Medellín, Colombia. Ingreso al Instituto Universitario de la Paz 
como docente para el año 2011 en donde actualmente ejerce. ORCID: 0000-0002-0335-7932

\section{Anexos}

Estrategias de Enseñanza -Aprendizaje basadas en los Modelos de Felder \& Soloman y Barriga \& Hernández.

\begin{tabular}{|c|c|c|c|c|}
\hline D & $\begin{array}{c}\text { ESTI } \\
\text { LO }\end{array}$ & $\begin{array}{l}\text { MODELO DE } \\
\text { FELDER }\end{array}$ & $\begin{array}{c}\text { ACTIVDA } \\
\text { DES } \\
\text { PROPUES } \\
\text { TAS POR } \\
\text { FELDER }\end{array}$ & ESTRATEGIAS \\
\hline \multirow{2}{*}{ 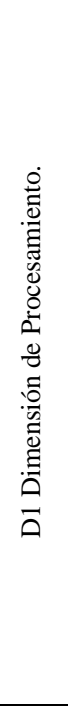 } & 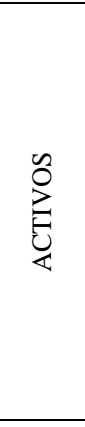 & $\begin{array}{l}\text { Tienen tendencia a } \\
\text { retener y entender } \\
\text { mejor la } \\
\text { información si } \\
\text { realizan alguna } \\
\text { actividad con ella. } \\
\text { "Let's try it out and } \\
\text { see how it works" }\end{array}$ & $\begin{array}{l}\text { Discusión, } \\
\text { Explicar a } \\
\text { otros } \\
\text { compañeros, } \\
\text { Trabajar en } \\
\text { grupos, } \\
\text { Resolución } \\
\text { de } \\
\text { Problemas }\end{array}$ & 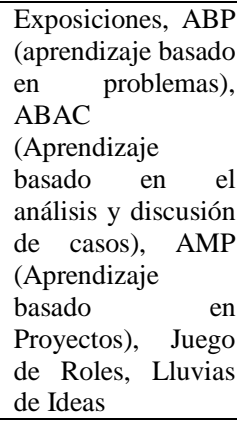 \\
\hline &  & $\begin{array}{l}\text { Prefieren pensar } \\
\text { acerca de la } \\
\text { información } \\
\text { primero "Let's } \\
\text { think it through } \\
\text { first" }\end{array}$ & $\begin{array}{l}\text { Análisis de } \\
\text { Lecturas, } \\
\text { Escribir } \\
\text { cortos } \\
\text { resúmenes, } \\
\text { notas de } \\
\text { clases con } \\
\text { tus propias } \\
\text { palabras }\end{array}$ & $\begin{array}{l}\text { Analogías, } \\
\text { Establecimiento de } \\
\text { relaciones } \\
\text { conceptuales, Juego } \\
\text { de Roles. }\end{array}$ \\
\hline \multirow[t]{2}{*}{ 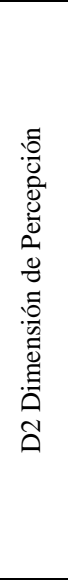 } & 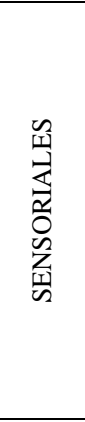 & $\begin{array}{l}\text { Prefieren hechos de } \\
\text { aprendizaje, les } \\
\text { gusta resolver } \\
\text { problemas ron } \\
\text { métodos bien } \\
\text { establecidos, son } \\
\text { más prácticos y } \\
\text { cuidadosos }\end{array}$ & $\begin{array}{l}\text { Conectar la } \\
\text { información } \\
\text { con la } \\
\text { realidad, Los } \\
\text { conceptos } \\
\text { aplicados a la } \\
\text { práctica, } \\
\text { Lluvias de } \\
\text { ideas. }\end{array}$ & $\begin{array}{l}\text { Exposiciones, ABP } \\
\text { (aprendizaje basado } \\
\text { en problemas), } \\
\text { ABAC } \\
\text { (Aprendizaje } \\
\text { basado en el } \\
\text { análisis y discusión } \\
\text { de casos), AMP } \\
\text { (Aprendizaje } \\
\text { basado en } \\
\text { Proyectos), Juego } \\
\text { de Roles, Lluvias } \\
\text { de Ideas }\end{array}$ \\
\hline & 葛。 & $\begin{array}{l}\text { A menudo prefieren } \\
\text { descubrir } \\
\text { posibilidades y } \\
\text { relacionarlas, les } \\
\text { gusta la innovación. }\end{array}$ & Lecturas & $\begin{array}{l}\text { Juego de } \text { Roles, } \\
\text { Lluvias de Ideas, } \\
\text { Análisis de Textos }\end{array}$ \\
\hline 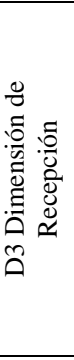 & 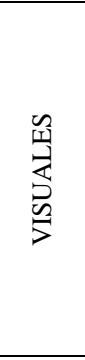 & $\begin{array}{l}\text { Recuerdan mejor lo } \\
\text { que ellos ven, } \\
\text { pinturas, } \\
\text { diagramas, } \\
\text { diagramas de flujos, } \\
\text { líneas de tiempo, } \\
\text { films y } \\
\text { demostraciones. }\end{array}$ & $\begin{array}{l}\text { Diagramas, } \\
\text { fotografías, } \\
\text { diagramas de } \\
\text { flujos, } \\
\text { bocetos, } \\
\text { esquemas y } \\
\text { otras } \\
\text { representaci } \\
\text { ones visuales } \\
\text { del material } \\
\text { del curso. } \\
\end{array}$ & $\begin{array}{l}\text { Organizadores } \\
\text { Gráficos en general, } \\
\text { Videos, } \\
\text { Diapositivas, } \\
\text { Salidas de Campo }\end{array}$ \\
\hline
\end{tabular}

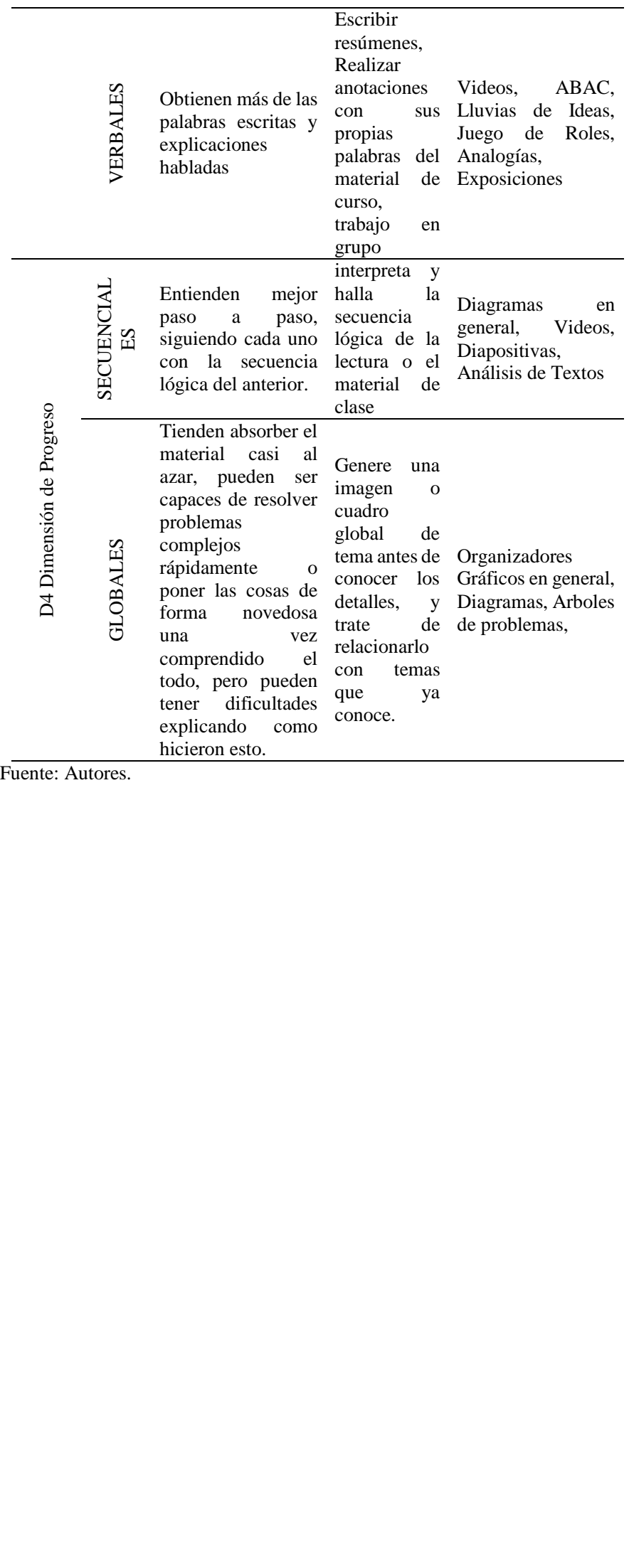

